\title{
Evaluation of azacitidine in patients with transplant-ineligible myelodysplastic syndromes and acute myeloid leukemia with myelodysplasia-related changes in a Japanese clinical setting
}

\author{
AYA NAKAYA, SHINYA FUJITA, ATSUSHI SATAKE, TAKAHISA NAKANISHI, \\ YOSHIKO AZUMA, YUKIE TSUBOKURA, RYO SAITO, AKIKO KONISHI, MASAAKI HOTTA, \\ HIDEAKI YOSHIMURA, KAZUYOSHI ISHII, TOMOKI ITO and SHOSAKU NOMURA
}

First Department of Internal Medicine, Kansai Medical University, Osaka 573-1010, Japan

Received February 17, 2019; Accepted June 26, 2019

DOI: $10.3892 / \mathrm{ol} .2019 .11225$

\begin{abstract}
Patients with high-risk myelodysplastic syndromes (MDS) treated with azacitidine (AZA) have exhibited improved overall survival. However, information on AZA in real-world settings is limited. The present study retrospectively analyzed 85 patients with MDS treated with AZA. Complete response was achieved in $24 \%$ of cases and hematologic improvement in 29\%. Severe adverse events (grade $\geq 3$ ) included neutropenia and infection. Multivariate analysis identified higher revised international prognostic scoring system (IPSS-R) and male sex as significant factors affecting survival. However, the present study did not identify any significant associations between patient characteristics and response to AZA. In conclusion, AZA could produce a hematologic response in $\sim 53 \%$ of patients with MDS. Furthermore, IPSS-R may reflect MDS prognosis. Further studies are required to establish the criteria for identifying patients likely to obtain maximum benefit from AZA treatment.
\end{abstract}

\section{Introduction}

Myelodysplastic syndromes (MDS) are hematopoietic stem cell disorders characterized by ineffective hematopoiesis and peripheral blood cytopenia. Degrees of cytopenia, increasing bone marrow blasts and cytogenetic abnormalities reflect the risk of progression to acute myelogenous leukemia, and are incorporated into prognostic scoring systems (1). The median age of diagnosis is 70-79 years (2). Therefore, patients in this age group are mostly ineligible for transplantation. Introduction

Correspondence to: Dr Aya Nakaya, First Department of Internal Medicine, Kansai Medical University 2-5-1 Shin-machi, Hirakata, Osaka 573-1010, Japan

E-mail: nakaya1016@yahoo.co.jp

Key words: myelodysplastic syndromes, azacytidine, revised international prognostic scoring system, transplant ineligible, real-world setting of a novel hypomethylating agent, azacitidine (AZA), caused a stir in MDS treatment strategy. High-risk MDS patients have shown improved overall survival (OS) when treated with the hypomethylating agent AZA compared with conventional therapies (1). A phase 1/2 study of AZA-7 in Japan demonstrated that AZA was effective, safe, and well tolerated in MDS patients (2). Based on these results, AZA was approved for MDS in all-risk groups in Japan in 2011, and has since become the first-line treatment for transplant-ineligible high-risk MDS patients. However, Japanese post-marketing data assessing the safety and efficacy of AZA in real-world settings are currently limited. We here report the results for patients with MDS patients treated with AZA in a real-world setting.

\section{Patients and methods}

Patients. In this retrospective study, transplant-ineligible patients with MDS were treated with AZA at two hematology centers, Kansai Medical University Hospital and Kansai Medical University Medical Center, between June 2012 and August 2018. MDS subtypes were defined according to the World Health Organization (WHO) 2016 criteria (3). Risks were assessed using the revised international prognosis scoring system (IPSS-R) (4). Patients who received a transplant were excluded. In our facility, indication for transplant is patient with high risk in IPSS-R, under 60 years old, without serious complications. This study was conducted in accordance with the Declaration of Helsinki and the requirements of the institution's review board.

Treatment regimens. Patients received one cycle of AZA treatment consisting of $75 \mathrm{mg} / \mathrm{m}^{2} /$ day for 5 or 7 days per month without any chemotherapy, according to phase III study ${ }^{1}$. Two types of alternative scheduling were used at the treatment sites because weekend treatment was not possible: a 5-day regimen consisting of 5 days of AZA treatment with no weekend treatment, and a 7-day regimen consisting of 5 days of AZA treatment with no weekend treatment, followed by 2 further days of AZA treatment. Patients with neutropenia received antimicrobial prophylaxis. The red blood cell and platelet transfusion thresholds were as follows: Hemoglobin level $<7 \mathrm{~g} / \mathrm{dl}$ and platelet count $<20 \times 10^{9} / 1$, respectively. We did not 
use granulocyte-colony stimulating factor (G-CSF) and other stimulating factors as a standard therapy.

Response criteria. Efficacy was measured using complete remission (CR), hematologic improvement (HI), stable disease (SD), and failure, as defined by the International Working Group (IWG) 2006 criteria (5).

However, not all patients in our cohort underwent bone marrow examination to evaluate response. We confirmed CR was patients who compatible to IMG criteria with evaluation by bone marrow exam, and $\mathrm{HI}$ included patients who compatible to IMG criteria without bone marrow evaluation. The timing of evaluation was various, so we evaluated best response.

Evaluation of safety. Toxicity was evaluated according to the Common Terminology Criteria for Adverse Events (AEs) (CTCAE4.0) [http://ctep.cancer.gov/protocolDevelopment/electronic_applications/ctc.htm\#ctc_40].

Statistical analysis. The primary end point is OS. OS was calculated from the start of AZA treatment until the time of death or the last clinical follow-up. Survival curves were generated using the Kaplan-Meier method, and differences were evaluated using the log-rank test. Multivariate Cox proportional hazards models were used to determine whether baseline characteristics were associated with OS. Associations between baseline characteristics and response were analyzed using Fisher's exact test. To determine if correction of cytopenia improved OS, a time-dependent Cox model was used to assess the prognostic impact of achieving CR and HI.

All statistical tests were two-sided, statistical significance was defined as $\mathrm{P}<0.05$, and $95 \%$ confidence intervals (CI) were calculated. All statistical analyses were performed using EZR (Saitama Medical Center, Jichi Medical University, Saitama, Japan), which is a graphical user interface for $\mathrm{R}$ version 2.13.0 (The R Foundation). Specifically, EZR is a modified version of $\mathrm{R}$ Commander (version 1.6-3), which adds statistical functions frequently used in biostatistics (6).

\section{Results}

Patient characteristics. The clinical characteristics of the patients ( $\mathrm{n}=85$; median age, 73 years, range, 50-95 years; $59 \%$ male) included in this study are shown in Table I. Patients were classified according to the 2016 WHO Classification as follows: Acute myeloid leukemia (AML) with myelodysplasia-related changes [27\%; the 2016 WHO classification for AML notably includes the French American British classification of MDS as refractory anemia with excess blasts in transformation (RAEB-T)], MDS with excess blasts type 1 (18\%), MDS with excess blasts type $2(8 \%)$, myelodysplastic/myeloproliferative neoplasm (16\%), therapy-related myeloid neoplasms (5\%), and others $(2 \%)$. The median number of AZA cycles was seven [standard deviation (SD): 2-54]. Forty-five percent of patients were treated using the 5-day regimen. The median follow-up period was 12.0 (SD: 1.2-73.6) months.

Treatment outcomes. CR was achieved in $24 \%$ of patients, HI in $29 \%$, and SD in 41 and $6 \%$ of patients had treatment failure according to the IWG criteria (Fig. 1A). In patients with HI, the specific responses of cytopenia in the three lineages were as follows: Erythroid (HI-E) in one patient, platelet response (HI-P) in 12 patients, and neutrophil response (HI-N) in three patients. Two-lineage responses were as follows: HI-E and HI-P in one patient, HI-P and HI-N in seven patients, and HI-E and HI-N in one patient (Fig. 1B). The median OS was 22.7 months (12.5-28.7). The median OS according to response was as follows: 24.9 months (12.4-not achieved) for both CR and HI and 15.9 (8.9-25.2) months for both SD and failure $(\mathrm{P}=0.146)$. The prognostic classification of MDS regarding survival was made using IPSS-R, and the median OS was as follows: Not available for very low and low, 31.6 months for intermediate, 23.0 months for high, and 12.0 months for very high $(\mathrm{P}=0.09)$ (Fig. 2).

Toxicity. Severe AEs (grade $\geq 3$ ) were neutropenia $(n=16)$ and infection $(n=16)$. Five patients died from severe infection. Other hematological AEs were thrombocytopenia $(n=8)$ and anemia $(n=5)$. Non-hematological AEs were grade 3 renal failure $(n=1)$ and grade 3 febrile neutropenia $(n=2)$.

Predictors for survival. We carried out multivariate analysis of OS to identify the clinical factors that defined patients who achieved improved outcomes after AZA treatment (Table II). We evaluated age $>75$ years, male sex, 7-day regimen, RAEB, and IPSS-R $\geq$ high. Male sex and IPSS-R $\geq$ high were significantly associated with OS [hazard ratio (HR): 1.97 (95\% CI: 1.20-3.81], $\mathrm{P}=0.043$; HR: 2.71 (95\% CI: 1.21-5.30), $\mathrm{P}=0.005$; respectively) (Table II).

Other factors including AEs dropped out after backward stepwise selection. We also performed Fisher's test to identify the basic characteristics of patients who responded. However, we failed to find any significant associations between patient characteristics and response to AZA (Table III).

Influence of HI on OS. To determine if correction of cytopenia improved OS, we assessed the prognostic impact of achieving CR and HI using a time-dependent Cox model (Table IV). However, we failed to find any significant associations between response and OS.

\section{Discussion}

In this retrospective study, we analyzed the outcomes of patients with all-risk transplant-ineligible MDS following treatment with AZA in a clinical setting. The results suggested that AZA administration was effective, with an overall response rate of $>50 \%$.

AZA has been studied in patients with higher-risk MDS in two major randomized multicenter trials, with median survivals of 21 months in CALGB9221 (7) and 24.5 months in AZA-001 (1). The median survival in the current study was comparable (22.7 months), even though our cohort included all-risk patients and more elderly patients than the trials. No reports to date have evaluated the effect of AZA using IPSS-R. Most patients in the current cohort were high- or intermediate-risk according to IPSS-R, and OS according to IPSS-R classification revealed that high-risk patients had poorer OS, though IPSS-R classification showed no difference in response to AZA using Fisher's exact analysis. This suggests 
Table I. Patient characteristics.

\begin{tabular}{lc}
\hline Variable & Value $(\mathrm{n}=85)$ \\
\hline Median age, years (range) & $73(50-95)$ \\
Male, \% & 59 \\
PS 0/1, $\%$ & 86 \\
Diagnosis, $\%$ & \\
MDS-RS & 18 \\
MDS-EB-1 & 24 \\
MDS-EB-2 & 8 \\
MDS/MPN & 16 \\
t-MN & 5 \\
AML with myelodysplasia-related changes & 27 \\
(RAEB-T in FAB) & \\
Others & 2 \\
IPSS-R, \% & \\
Very low & 2 \\
Low & 11 \\
Intermediate & 29 \\
High & 31 \\
Very high & 27 \\
Median cycle, $n$ (range) & $72.0(1.2-73.6)$ \\
5 days regimen, \% & \\
Median follow-up periods, months (range) & \\
\hline
\end{tabular}

PS, performance status; MDS-RS, myelodysplastic syndromes with ring sideroblasts; MDS-EB, myelodysplastic syndromes with excess blasts; MDS/MPN, myelodysplastic/myeloproliferative neoplasms; t-MN, therapy-related myeloid neoplasms; AML, acute myeloid leukemia; RAEB-T, refractory anemia with excess blasts in transformation; FAB, French American British classification; IPSS-R, revised international prognosis scoring system.

that IPSS-R reflects the outcome of MDS but not the efficacy of AZA treatment, i.e., the prognosis of MDS is largely related to chromosomal abnormalities, but treatments are not.

More patients who achieved HI also achieved platelet recovery, suggesting that the effects of AZA might be predicted by platelet recovery. van der Helm et al (8) reported that platelet doubling after the first cycle of AZA might be a useful indicator of AZA efficacy. In our study, the median number of AZA cycles required to achieve $\mathrm{HI}$ was three, suggesting that early platelet recovery may be a useful predictor of response. However, there were few responders in terms of erythroid recovery. AZA was approved for all-risk MDS in Japan in 2011, before the approval of erythropoiesis-stimulating agents (ESAs) for low-risk MDS. Unlike in western countries, some patients therefore initially received AZA without ESA, which might affect the erythroid recovery response in Japan. If ESAs were used prior to AZA, the erythroid recovery may have been different. We assessed the influence of HI on OS but failed to find any significant associations between response and OS. However, we think the most benefit of using AZA is reduction of number of transfusions. We did not assess the quality of life (QOL) improvement, however, reduction of number of transfusions might have improved patient's QOL.

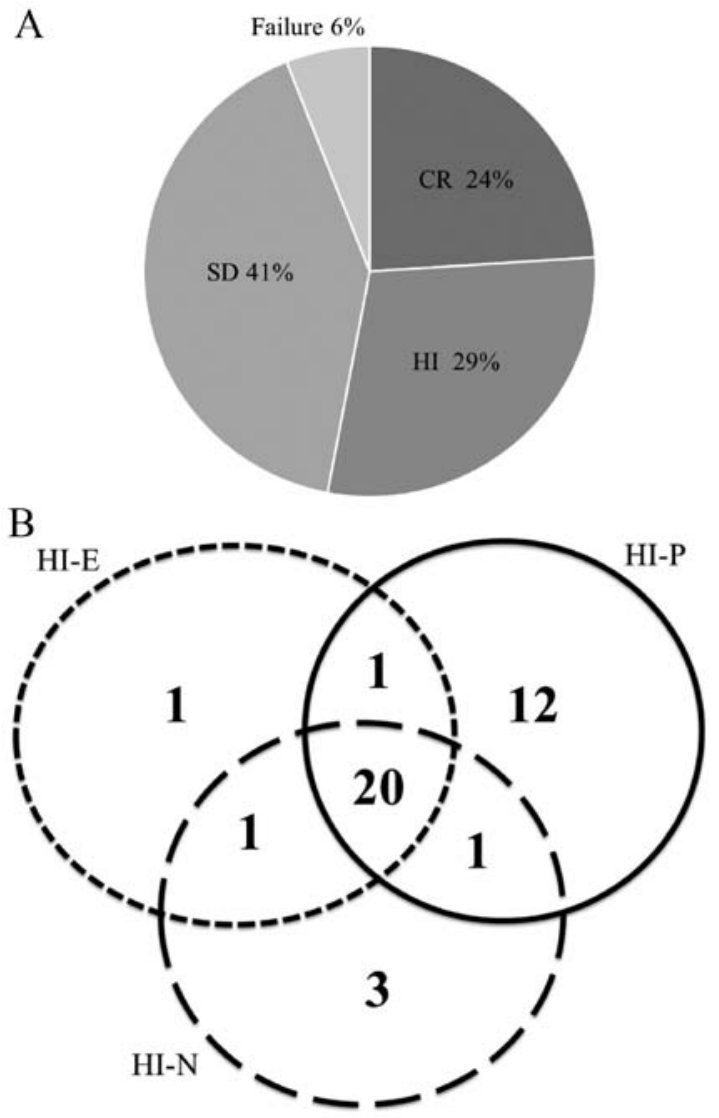

Figure 1. Treatment outcomes. (A) Response to azacytidine. CR was achieved in $24 \%$ of patients, HI was achieved in $29 \%$ of patients, $41 \%$ of patients had $\mathrm{SD}$, and $6 \%$ of patients had failure according to the International Working Group criteria. (B) Specific responses of cytopenia in the three lineages. CR, complete response; HI, hematologic improvement; SD, stable disease; HI-E, hematologic improvement in erythroid; HI-P, hematologic improvement in platelet; HI-N, hematologic improvement in neutrophil.

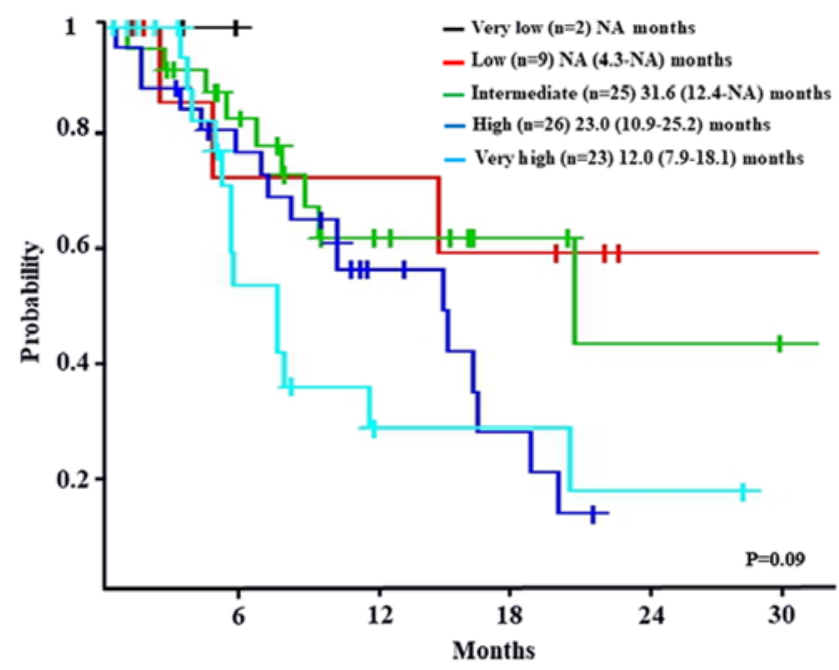

Figure 2. Median overall survival time according to IPSS-R. The P-value shown is the value obtained when testing the null hypothesis that all five groups have the same survival rate. NA, not available.

The recommended schedule for AZA administration is $75 \mathrm{mg} / \mathrm{m}^{2}$ for 7 consecutive days every 28 days. However, a 7 -consecutiv day regimen is difficult to administer in the 
Table II. Multivariate analysis for overall survival.

\begin{tabular}{lccc}
\hline Variables & Hazard ratio & $95 \% \mathrm{CI}$ & P-value \\
\hline Male & 1.97 & $1.20-3.81$ & 0.043 \\
Female & 1 & & \\
IPSS-R ( $\geq$ high $)$ & 2.71 & $1.21-5.30$ & 0.005 \\
IPSS-R $(<$ high $)$ & 1 & &
\end{tabular}

IPSS-R, revised international prognosis scoring system.

Table III. Results of Fisher's exact test investigating the association of variables with the response to AZA.

\begin{tabular}{lccc}
\hline Variables & Odds ratio & $95 \% \mathrm{CI}$ & P-value \\
\hline Age $\geq 75$ years & 1.09 & $0.44-2.72$ & 0.854 \\
Age $<75$ years & 1 & & \\
Male & 1.22 & $0.50-3.01$ & 0.667 \\
Female & 1 & & \\
7 day regimen & 0.70 & $0.28-1.74$ & 0.437 \\
5 day regimen & 1 & & \\
IPSS-R ( $\geq$ high) & 0.57 & $0.20-1.63$ & 0.291 \\
IPSS-R (<high) & 1 & & \\
RAEB & 2.41 & $0.85-6.79$ & 0.097 \\
$\begin{array}{l}\text { Not RAEB } \\
\text { AE positive }\end{array}$ & 1 & & \\
AE negative & 0.56 & $0.23-1.38$ & 0.207 \\
\hline
\end{tabular}

IPSS-R, revised international prognosis scoring system; RAEB, refractory anemia with excess blasts; $\mathrm{AE}$, adverse events.

Table IV. Time-dependent Cox model of overall survival according to the achievement of CR and HI.

\begin{tabular}{lccc}
\hline Variable & Hazard ratio & $95 \% \mathrm{CI}$ & P-value \\
\hline $\mathrm{CR}$ & 0.70 & $0.27-1.83$ & 0.465 \\
$\mathrm{HI}$ & 2.58 & $1.19-5.58$ & 0.016
\end{tabular}

CR, complete remission; HI, hematological improvement.

clinic because weekend administration is often not possible. Our study sites thus adopted alternative schedules of either 5- or 7-day regimens. There was no significant difference in efficacy between these two regimens according to univariate analysis, multivariate analysis for survival, or Fisher's exact test for response.

Despite numerous studies, the optimal schedule of AZA administration is currently unclear. Fujimaki et al (9) reported a retrospective study in which $52 \%$ of patients (high-risk MDS) achieved HI with the 5-day schedule. Morita et al (10) reported a phase 2 study in low-risk MDS patients in which $47.1 \%$ of patients achieved $\mathrm{HI}$ and $21.6 \%$ achieved CR with the 5-day regimen. Lyons et al (11) compared three different regimens (5 day, 5-2-2 days, and 5-2-5 day), and reported no significant difference in response. Although it is too early to draw conclusions regarding the optimal treatment regimen, current data suggest that the regimen length may not significantly affect prognosis.

We analyzed the basic characteristics of the patients in relation to response to AZA using Fisher's exact test, but failed to find any significant associations between patient characteristics and AZA treatment response. The patient characteristics predicting response to AZA thus remain unknown. We also identified the baseline characteristics associated with survival by multivariate analysis. It revealed that male sex and a high-risk IPSS-R classification had high HRs for survival. As we described above, this suggests that IPSS-R reflects the prognosis of MDS but not the efficacy of AZA treatment. Lifestyle and habits such as tobacco and alcohol have been reported to increase the prevalence of MDS, which may contribute to the worse prognosis of men (12).

In our study, five patients died of infection after AZA treatment. None of these patients showed a response, all had stable disease, and all developed neutropenia due to AZA. In practice, some patients continue AZA even if they do not show a response. The guidelines recommend that, in the absence of any alternative, transplant-ineligible patients should continue AZA until progression, potentially resulting in unplanned treatment and subsequent neutropenia. We suggest that this mortality rate may thus reflect the real-world setting. Beguin et al (13) reported that six of 99 patients died of AEs, though they did not describe the AEs in detail.

This study had some limitations, including its retrospective design, the small number of study sites, and small sample size. In this study, we excluded transplant eligible patients, considering that it might be difficult to evaluate the effectiveness of AZA alone. Conversely, it may include worse conditioned patients who cannot undertake transplant, that might become the selection bias.

According to the National Comprehensive Cancer Network guidelines for low-risk MDS (14), AZA treatment is recommended after failure of ESA treatment. As previously stated however, AZA was approved prior to ESA in Japan, and some patients in our study thus received AZA without prior ESA treatment. The 2016 WHO classification of AML with myelodysplasia-related changes includes MDS as RAEB-T in the FAB classification. In clinical practice, patients belonging to this category are treated with AZA, and we therefore included this group in our study. Not all patients in our cohort underwent bone marrow examination to evaluate response, and we could therefore not judge 'partial remission' or 'marrow CR', which require precise bone marrow blast counts. We therefore used CR, HI, SD, and failure to evaluate outcomes in this study. Furthermore, the timing of evaluation was various, and it depended on physicians' decision. We picked up the best response retrospectively. This is because there is no criteria of evaluation of AZA. We are not certain when AZA shows the effectiveness. This point needs to be clarified in the prospective trials.

In conclusion, the results of this study suggest that all-risk transplant-ineligible MDS patients may benefit from AZA treatment. IPSS-R risk category may not reflect the efficacy of 
AZA, but may predict overall prognosis in patients with MDS. AZA can be administered safely to MDS patients. However, further studies are warranted to establish the criteria for identifying patients likely to obtain maximum benefit from AZA treatment, and to develop optimal treatment strategies.

\section{Acknowledgements}

Not applicable.

\section{Funding}

No funding was received.

\section{Availability of data and materials}

The datasets used and/or analyzed during the current study are available from the corresponding author on reasonable request.

\section{Authors' contributions}

AN and SN designed the study. AN performed statistical analysis and interpretation of data, and drafted the article. AN, SF, AS, TN, YT, RS, YA, AK, MH, HY, KI, TI and SN performed patient treatment, provided patient data, and analyzed and interpreted it. AN and SN drafted the article and revised it critically for important intellectual content. AN, SF, AS, TN, YT, RS, YA, AK, MH, HY, KI, TI and SN provided final approval of the version to be submitted. All authors read and approved the final manuscript.

\section{Ethics approval and consent to participate}

The present study was conducted in accordance with the Declaration of Helsinki and the requirements of the institution's review board.

\section{Patient consent for publication}

Not applicable.

\section{Competing interests}

The authors declare that they have no competing interests.

\section{References}

1. Fenaux P, Mufti GJ, Hellstrom-Lindberg E, Santini V, Finelli C, Giagounidis A, Schoch R, Gattermann N, Sanz G, List A, et al: Efficacy of azacitidine compared with that of conventional care regimens in the treatment of higher-risk myelodysplastic syndromes: A randomised, open-label, phase III study. Lancet Oncol 10: 223-232 2009.

2. Uchida T, Ogawa Y, Kobayashi Y, Ishikawa T, Ohashi H, Hata T, Usui N, Taniwaki M, Ohnishi K, Akiyama H, et al: Phase I and II study of azacitidine in Japanese patients with myelodysplastic syndromes. Cancer Sci 102: 1680-1686, 2011.
3. Arber DA, Orazi A, Hasserjian R, Thiele J, Borowitz MJ, Le Beau MM, Bloomfield CD, Cazzola M and Vardiman JW: The 2016 revision to the World Health Organization classification of myeloid neoplasms and acute leukemia. Blood 127: 2391-2406, 2016.

4. Greenberg PL, Tuechler H, Schanz J, Sanz G, Garcia-Manero G, Solé F, Bennett JM, Bowen D, Fenaux P, Dreyfus F, et al: Revised international prognostic scoring system for myelodysplastic syndromes. Blood 120: 2454-2465, 2012.

5. Cheson BD, Greenberg PL, Bennett JM, Lowenberg B Wijermans PW, Nimer SD, Pinto A, Beran M, de Witte TM, Stone RM, et al: Clinical application and proposal for modification of the International Working Group (IWG) response criteria in myelodysplasia. Blood 108: 419-425, 2006.

6. Kanda Y: Investigation of the freely available easy-to-use software 'EZR' for medical statistics. Bone Marrow Transplant 48: 452-458, 2013.

7. Silverman LR, Demakos EP, Peterson BL, Kornblith AB Holland JC, Odchimar-Reissig R, Stone RM, Nelson D, Powell BL, DeCastro CM, et al: Randomized controlled trial of azacitidine in patients with the myelodysplastic syndrome: A study of the cancer and leukemia group B. J Clin Oncol 20: 2429-2440, 2002.

8. van der Helm LH, Alhan C, Wijermans PW, van Marwijk Kooy M, Schaafsma R, Biemond BJ, Beeker A, Hoogendoorn M, van Rees BP, de Weerdt $\mathrm{O}$, et al: Platelet doubling after the first azacitidine cycle is a promising predictor for response in myelodysplastic syndromes (MDS), chronic myelomonocytic leukaemia (CMML) and acute myeloid leukaemia (AML) patients in the Dutch azacitidine compassionate named patient programme, Br J Haematol 155: 599-606, 2011.

9. Fujimaki K, Miyashita K, Kawasaki R and Tomita N: Efficacy and safety of a 5-day regimen of azacitidine for patients with high-risk myelodysplastic syndromes, Eur J Haematol 97: 228-231, 2016.

10. Morita Y, Maeda Y, Yamaguchi T, Urase F, Kawata S, Hanamoto H, Tsubaki K, Ishikawa J, Shibayama H, Matsumura I and Matsuda M: Five-day regimen of azacitidine for lower-risk myelodysplastic syndromes (refractory anemia or refractory anemia with ringed sideroblasts): A prospective single-arm phase 2 trial. Cancer Sci 109: 3209-3215, 2018.

11. Lyons RM, Cosgriff TM, Modi SS, Gersh RH, Hainsworth JD, Cohn AL, McIntyre HJ, Fernando IJ, Backstrom JT and Beach CL: Hematologic response to three alternative dosing schedules of azacitidine in patients with myelodysplastic syndromes, J Clin Oncol 27: 1850-1856. 2009.

12. Ugai T, Matsuo K, Sawada N, Iwasaki M, Yamaji T, Shimazu T, Sasazuki S, Inoue M, Kanda Y and Tsugane S; Japan Public Health Centre-based Prospective Study Group: Smoking and alcohol and subsequent risk of myelodysplastic syndromes in Japan: The Japan Public Health Centre-based Prospective Study. Br J Haematol 178: 747-755, 2017.

13. Beguin Y, Selleslag D, Meers S, Graux C, Bries G, Deeren D, Vrelust I, Ravoet C, Theunissen K, Voelter V, et al: Safety and efficacy of azacitidine in Belgian patients with high-risk myelodysplastic syndromes, acute myeloid leukaemia, or chronic myelomonocytic leukaemia: results of a real-life, non-interventional post-marketing survey. Acta Clin Belg 70: 34-43, 2015.

14. The NCCN Clinical Practice Guidelines in Oncology, Myelodysplastic Syndromes, Version2.20 2019-October18, 2018 https://www.nccn.org/professionals/physician_ gls/pdf/mds.pdf. Accessed November 11, 2018.

This work is licensed under a Creative Commons Attribution-NonCommercial-NoDerivatives 4.0 International (CC BY-NC-ND 4.0) License. 